\title{
Resultados del primer registro chileno de angina inestable: características clínicas, perfil de riesgo y tratamiento
}

\author{
Ramón Corbalán H, Carolina Nazzal Na, Germán Eggers R, \\ Jaime Bartolucci J, Juan Carlos Prieto D, Milton Alcaíno I, \\ Benjamín Stockins F, Pabla Campos P, Alejandro Dapelo A. \\ En representación del G rupo de Angina Inestable, \\ Departamento de Estudios Multicéntricos, Sociedad Chilena de \\ Cardiología y Cirugía Cardiovascular.
}

\section{Results of the first Chilean registry of unstable angina. Clinical features, risk profile and treatment}

\begin{abstract}
Background: From January 2000 to June 2002, the first Chilean registry of unstable angina was carried out, with the participation of 15 hospitals. Aim: To report the clinical and demographic features of 600 patients with unstable angina, their risk profile and prognosis. Material and methods: The inclusion criteria for this prospective registry were a history of recent onset of chest pain ( $<48$ hours) or a change in the character of previous angina, associated to ischemic electrocardiographic changes and/or positive markers of myocardial damage. Results: Mean age of the patients was 65 years and 37\% were women. Among coronary risk factors, $63 \%$ had hypertension, $27 \%$ had diabetes, $52 \%$ had dyslipidemia, $31 \%$ smoked and $21 \%$ had a family history of atherosclerosis. On admission $94 \%$ of patients had chest pain, associated to ST segment depression in 44\%, negative T waves in $28 \%$ and positive markers of myocardial damage in $30 \%$. Fifty seven percent received intravenous nitroglycerin, $47 \%$ received oral nitrates, $69 \%$ beta blockers and $15 \%$, calcium antagonists. Antithrombotic therapy included aspirin in $96 \%$, heparin in $74 \%$, ticlodipine or clopidogrel in $19 \%$ and IIb/IIIa inhibitors in $12 \%$. A coronary angiogram was performed in $52 \%$, angioplasty in $25 \%$ and coronary bypass surgery in $13 \%$. Hospital mortality was $2.6 \%$. The incidence of new ischemic events was: myocardial infarction in $2.8 \%$ recurrent ischemia in $9.5 \%$ and refractory ischemia in $2 \%$. The incidence of adverse events increased according to a higher risk profile. Conclusions: The demographic and clinical features, treatment and mortality of these patients are similar to those reported in international registries, with a low mortality rate (Rev Méd Chile 2004; 132: 135-43).

(Key Words: Angina, unstable; Angioplasty, transluminal, percutaneous coronary; Coronary artery bypass; Coronary disease)
\end{abstract}

Recibido el 1 de julio, 2003. Aceptado en versión corregida el 2 de diciembre, 2003.

a EU Magíster en Salud Pública (c)

Correspondencia a: Dr. Ramón Corbalán. Hospital Clínico de la Universidad Católica de Chile. Marcoleta 367, Piso 6.

Fax 6392037. E mail: corbalan@med.puc.cl. 
L a angina inestable y el infarto del miocardio sin elevación del segmento ST constituyen las manifestaciones clínicas más frecuentes de la enfermedad coronaria. Por tener una fisiopatología común, ambas afecciones se engloban dentro del mismo contexto clínico. El rápido avance en el conocimiento de los síndromes coronarios agudos, ha sido determinante para que las recomendaciones para el diagnóstico y tratamiento de esta afección se hayan renovado constantemente, tanto en Europa como en Estados Unidos ${ }^{1,2}$. Al mismo tiempo, se han realizado una gran cantidad de ensayos clínicos para evaluar nuevas estrategias terapéuticas y se han utilizado distintos esquemas para la estratificación de riesgo y optimización acorde del tratamiento.

En Chile se han publicado, hasta la fecha, dos pautas de recomendaciones terapéuticas de la angina inestable e infarto no $\mathrm{Q}^{3,4}$. Sin embargo, no hay información disponible sobre cómo se están cumpliendo estas pautas ni tampoco sobre cuál es la realidad de esta afección en nuestro medio. Por esta razón, se constituyó un grupo de trabajo en el Departamento de Estudios Multicéntricos de la Sociedad Chilena de Cardiología y Cirugía Cardiovascular para llevar a cabo un registro nacional de angina inestable e infarto del miocardio sin elevación del segmento ST.

En el presente trabajo damos cuenta de los resultados obtenidos con el registro de 600 pa- cientes con angina inestable, efectuado en distintas instituciones de salud en Chile.

\section{MATERIAL Y MÉTODO}

El Registro Nacional de Síndromes Coronarios Agudos es un estudio prospectivo, observacional, de pacientes ingresados con diagnóstico de angina inestable o infarto agudo del miocardio sin supradesnivel del segmento ST (SDST), iniciado en enero de 2000. Una red de 15 hospitales participantes enrolaron pacientes en forma consecutiva durante un período de dos años; en junio de 2002 habían sido incorporados 600 pacientes. En la Tabla 1 se muestra la lista de centros hospitalarios que participaron en el registro, el número de pacientes registrado en cada centro y cuáles de ellos disponen de laboratorio de hemodinamia y servicio de cirugía cardíaca.

En una ficha especialmente diseñada se registraron para cada paciente las variables demográficas, antecedentes cardiovasculares previos, factores de riesgo coronario, características del episodio de dolor que motivó el ingreso, alteraciones del electrocardiograma (SDST, infradesnivel del segmento ST (IDST), T negativa), marcadores de daño miocárdico, incluyendo valores de CK, CK-MB o troponinas. Se recolectó la información acenca del tratamiento farmacológico previo al ingreso, durante la hospitali-

Tabla 1.

\begin{tabular}{|lccc|}
\hline Hospitales & № Pacientes & Hemodinamia & C Cardiovascular \\
\hline Hospital Regional Arica & 5 & - & - \\
Hospital de La Serena & 5 & - & - \\
Hospital Quillota & 17 & - & - \\
Hospital Gustavo Fricke & 37 & Sí & Sí \\
Hospital Dipreca & 50 & Sí & Sí \\
Hospital JJ Aguirre & 53 & Sí & Sí \\
Hospital Asistencia Pública & 9 & Sí & Sí \\
Hospital Universidad Católica & 213 & Sí & Sí \\
Hospital Regional Concepción & 21 & Sí & - \\
Hospital Naval Talcahuano & 17 & - & - \\
Hospital Victoria & 3 & Sí & Sí \\
Hospital Regional Temuco & 17 & - & - \\
Hospital Regional Osorno & 11 & - & - \\
Hospital Regional Valdivia & 56 & - & - \\
Hospital Regional Puerto Montt & 86 & & \\
\hline
\end{tabular}


zación y el tratamiento con que fue dado de alta el paciente. Al mismo tiempo se realizó un seguimiento telefónico a los 6 meses, en cada institución participante, lo que se consiguió en $58 \%$ de los pacientes que habían completado el período.

Fueron criterios de inclusión la presencia de al menos dos de las siguientes características: dolor anginoso típico en las últimas $48 \mathrm{~h}$ previas al ingreso (nuevo o cambio de carácter de angina previa), alteraciones electrocardiográficas de tipo isquémico al momento del ingreso o marcadores de daño miocárdico positivos.

Se excluyeron pacientes con antecedentes de angioplastia o cirugía coronaria en los últimos 6 meses, enfermedad cardíaca concomitante tales como miocardiopatía dilatada o hipertrófica, valvulopatías y enfermedades sistémicas concomitantes que pudieran provocar aumento del trabajo cardíaco (infecciones, anemia, hipertiroidismo, etc).

Variables clínicas analizadas:

1. Antecedentes: Se analizaron los antecedentes cardiovasculares previos (coronarios, insuficiencia cardíaca, accidente vascular cerebral, enfermedad arterial periférica) y los factores de riesgo coronario (hipertensión arterial, dislipidemia, diabetes, tabaquismo, historia familiar). Se consideró la historia familiar cuando existían antecedentes de enfermedad coronaria en uno de los padres o hermanos del paciente.

2. Características del evento índice: Episodio de dolor más intenso en las últimas $48 \mathrm{~h}$, tiempo transcurrido desde el inicio de los síntomas al ingreso y número de episodios de más de 5 min de duración en las $24 \mathrm{~h}$ previas.

3. Características del electrocardiograma de ingreso, a las $24 \mathrm{~h}$ y al alta, en que se analizó la presencia de IDST $>0,5 \mathrm{~mm}$ en dos 0 más derivaciones electrocardiográficas continuas, SDST transitorio $>0,5 \mathrm{~mm}$, onda $\mathrm{T}$ negativa mayor de $3 \mathrm{~mm}$ y desarrollo de nueva onda $\mathrm{Q}$ o nuevo bloqueo de rama izquierda.

4. Marcadores de daño miocárdico: Se tomaron muestras de sangre al ingreso, a las 6 y $12 \mathrm{~h}$ de evolución para determinaciones de CK total, CK-MB o troponina I. La CK total y CK-MB se determinaron en 554 pacientes $(92,5 \%)$ y la troponina I en 230 pacientes $(38,4 \%)$. Se registró el límite normal alto para cada laboratorio participante.
Tratamiento: Se registró el empleo de medicamentos antiisquémicos, antitrombóticos y otros que recibieron los pacientes previo al ingreso, durante la hospitalización y al momento del alta. En la terapia antiisquémica se consignó el uso de nitratos por vía intravenosa u oral, bloqueadores beta adrenérgicos y antagonistas del calcio. Como terapia antitrombótica se registró el uso de aspirina, heparina no fraccionada o de bajo peso molecular, inhibidores plaquetanios IIb-IIIa, anticoagulantes orales y empleo de ticlopidina o clopidrogel. En otros medicamentos se consideró el empleo de estatinas, diuréticos, inhibidores de la enzima de conversión y amiodarona.

También se registró la realización de coronariografía durante la hospitalización, en cuyo caso se consideró como lesión coronaria crítica una estenosis igual o mayor al $70 \%$ y se precisó la arteria culpable del episodio. Se analizó también la realización de procedimientos de revascularización miocárdica (cirugía comnaria, angioplastia con y sin uso de stent), los que a su vez se clasificaron como procedimientos electivos o de urgencia.

Evolución intrahospitalaria: Se consignó el desarrollo de nuevos eventos cardíacos como isquemia recurrente, isquemia refractaria, infarto del miocardio y la mortalidad.

La isquemia recurrente fue definida como aquella que se presenta en la primeras 48 a $72 \mathrm{~h}$ de ingresado el paciente, caracterizada por un episodio anginoso, asociado a cambios electrocardiográficos transitorios, cediendo a la administración de nitratos. La isquemia refractaria se definió como angina prolongada de más de 20 min o 2 episodios consecutivos de angina de $10 \mathrm{~min}$ de duración, asociada a cambios transitorios del ST-T o a edema pulmonar o hipotensión arterial, que obliga a soporte hemodinámico y estudio angiográfico de urgencia. Finalmente, el infarto del miocardio se definió como una exacerbación prolongada de la angina de pecho, asociada a la aparición de nuevas ondas Q en el electrocardiograma o elevación enzimática característica. Los pacientes fueron controlados a los 6 meses, consignando la sobrevida, nuevas hospitalizaciones e intervenciones. Los que no acudieron a control fueron contactados telefónicamente.

Estratificación de riesgo: Para la estratificación de riesgo se utilizó el puntaje TIMI $^{5}$ que va de 0 a 7 
puntos, según las siguientes características: edad $\geq 65$ años, 3 ó más factores de riesgo coronario, antecedentes de enfermedad coronaria previa, uso de aspirina en los 7 días previos al ingreso, angina severa reciente $(<24 \mathrm{~h})$, desviación del segmento $\mathrm{ST} \geq 0,5 \mathrm{~mm}$ en 20 más derivaciones contiguas y elevación de marcadores de daño miocárdico.

Los pacientes fueron agrupados en 3 grupos: a) Grupo de bajo riesgo, que incluyó aquellos pacientes con 0 a 2 puntos, b) grupo de riesgo intermedio con 3 y 4 puntos, y c) grupo de alto riesgo, aquellos con 5 a 7 puntos. Los eventos cardiovasculares adversos intrahospitalarios, así como los que ocurrieron dentro de los primeros 6 meses, fueron analizados de acuerdo a esta estratificación de riesgo.

Estadística: Se utilizó el sistema SAS (Statistical Analysis System). Las variables categóricas se han descrito como porcentajes y las continuas utilizando los valores promedio \pm desviación estándar. Para determinar las diferencias entre los tres grupos de riesgo se utilizó el test de chi cuadrado y los resultados se expresan mediante su OR y 95\% de CI.

\section{Resultados}

En la Tabla 2 se muestran las características demográficas de los pacientes, la presencia de antecedentes cardiovasculares de la población y la prevalencia de los factores de riesgo, donde destacan la hipertensión arterial y la dislipidemia.
En relación a las características clínicas, al ingreso del paciente al centro hospitalario, el 93\% se presentó con dolor anginoso característico.

La edad promedio fue $65 \pm 12$ años. La distribución por edad y sexo se ilustra en la Figura 1. Se observa que $48 \%$ de las mujeres eran

\section{Tabla 2. C aracterísticas demográficas y clínicas de los pacientes}

\begin{tabular}{lr}
\hline Edad (años) & $64,7 \pm 12$ \\
Género femenino & $37,0 \%$ \\
Antecedentes cardiovasculares & \\
Angioplastia previa & $8,0 \%$ \\
Infarto previo & $23,2 \%$ \\
Cirugía de revascularización previa & $8,6 \%$ \\
Enfermedad venosa periférica & $8,8 \%$ \\
Angor crónico previo & $33,0 \%$ \\
Insuficiencia cardíaca previa & $10,2 \%$ \\
Factores de riesgo & \\
Hipertensión arterial & $62,7 \%$ \\
Diabetes & $26,4 \%$ \\
Dislipidemia & $52,4 \%$ \\
Tabaquismo & $31,5 \%$ \\
Antecedentes familiares de cardiopatía coronaria & $21,0 \%$ \\
Características al ingreso & \\
Horas desde inicio de síntomas e ingreso & $11,8 \pm 22$ \\
Sólo un episodio de dolor previo al ingreso & $71,0 \%$ \\
Angor al ingreso & $94,3 \%$ \\
IDST o SDST al ingreso & $44,2 \%$ \\
T negativa & $28,2 \%$ \\
Marcadores de daño positivos & $30,0 \%$ \\
$\quad$ CK de ingreso & $166 \pm 174$ \\
CK a las 24 h & $255 \pm 398$
\end{tabular}

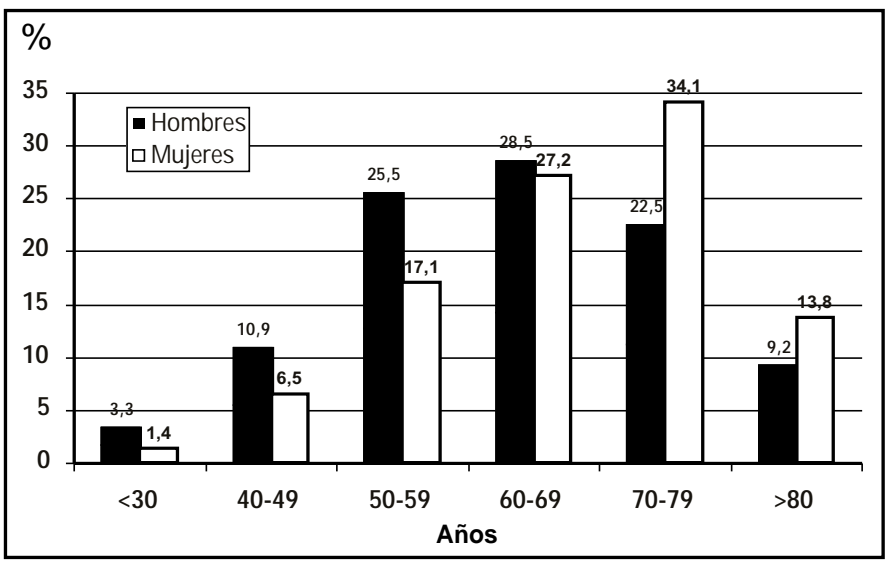

FiguRa 1. Distribución de pacientes según rangos de edad y sexo. 
mayores de 70 años, porcentaje que alcanza sólo $32 \%$ en los hombres. Los menores de 40 años corresponden a $2,6 \%$ de los pacientes.

El análisis de los factores de riesgo coronario mostró que éstos fueron más prevalentes en la mujeres, siendo significativamente mayor esta diferencia para la hipertensión arterial y la diabetes. El tabaquismo, en cambio, fue más prevalente entre los hombres (Figura 2).

En la Figura 3 se muestra la distribución de los pacientes de acuerdo al puntaje de riesgo TIMI, observándose que la mayoría se encuentra entre los 2 y 4 puntos, mientras que sólo $2 \%$ alcanza el puntaje máximo. La distribución por grupos fue de $29,9 \%$ en bajo riesgo, $47,6 \%$ en riesgo intermedio y $22,5 \%$ en riesgo alto.

La Tabla 3 muestra la terapia farmacológica utilizada durante la hospitalización y al alta. En la terapia antitrombótica de la fase aguda el empleo de aspirina fue superior a 95\% y más de $70 \%$ de
Tabla 3. Tratamiento intrahospitalario y al alta de los pacientes

\begin{tabular}{|lcc|}
\hline & $\begin{array}{c}\text { Intrahospitalario } \\
\%\end{array}$ & $\begin{array}{c}\mathrm{Al} \text { alta } \\
\%\end{array}$ \\
\hline Terapia antitrombótica & & \\
$\quad$ Aspirina & 96 & 74 \\
Heparina IV & 30,5 & - \\
Heparina bajo peso & 44 & 1 \\
Ticlopidina & 23 & 18 \\
Terapia antiisquémica & & \\
ß Bloqueadores & 69 & 47 \\
Nitratos iv & 57 & - \\
Nitratos orales & 47 & 31 \\
Antagonistas del calcio & 15 & 12 \\
Inhibidores & 35 & 37 \\
Otros & & \\
Estatinas & 37 & 31 \\
\hline
\end{tabular}

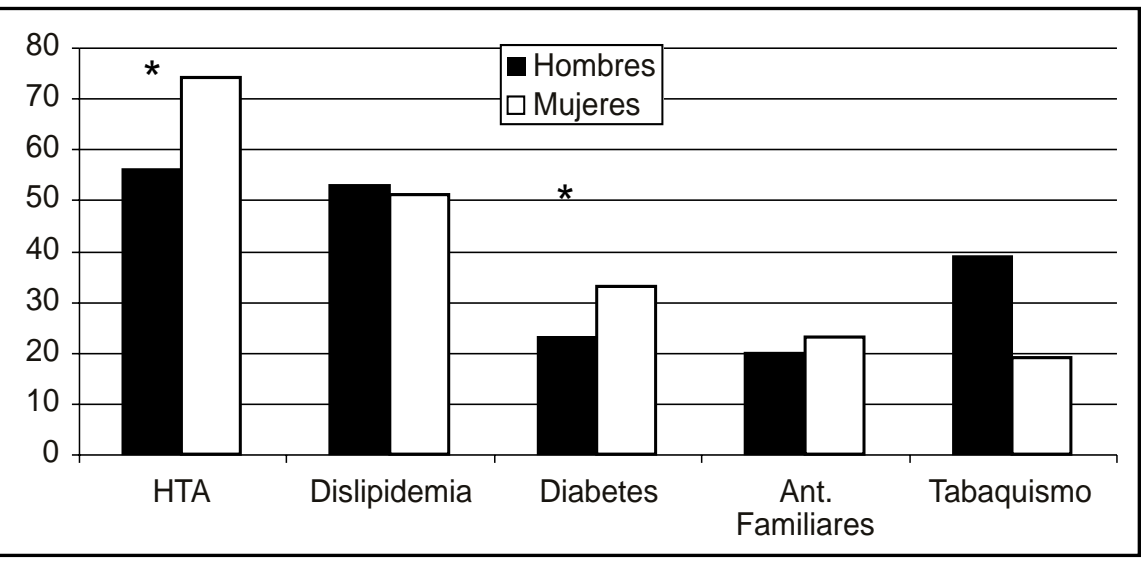

Figura 2. Distribución de pacientes según factores de riesgo y sexo.

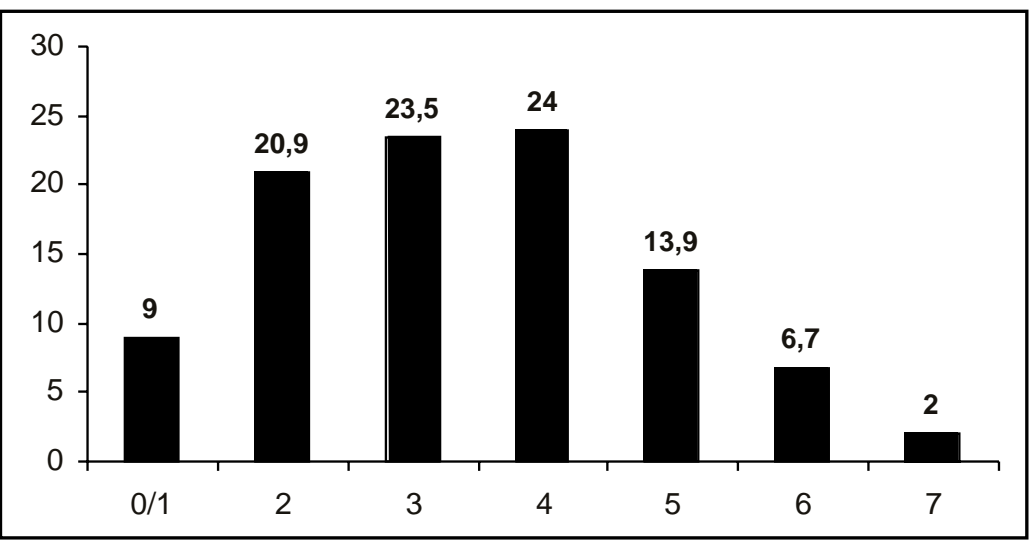

FIgURA 3. Distribución de pacientes según puntaje TIMI. 
los pacientes recibió alguna forma de heparina. En cuanto a la terapia antiisquémica, los medicamentos más utilizados por vía oral fueron los bloqueadores beta adrenérgicos y los nitratos. Se observa que al momento del alta se indicó estatinas a más de $30 \%$ de los pacientes.

En la Tabla 4 se muestra el empleo de coronariografía y procedimientos de revascularización miocárdica intrahospitalarios. En esta serie, más de $50 \%$ de los pacientes fue estudiado con coronariografía y los procedimientos de revascularización miocárdica se efectuaron en más de 30\% de los casos, independientemente de la estratificación de riesgo. En el grupo de riesgo alto se constató un porcentaje significativamente mayor de cirugía coronaria de urgencia.

La mortalidad intrahospitalaria global fue de $2,6 \%$, la que varió en forma significativa de acuerdo al puntaje de riesgo TIMI. La incidencia de nuevos eventos isquémicos intrahospitalarios fue de: infarto del miocardio $2,8 \%$, isquemia recurrente $9,5 \%$ e isquemia refractaria $2,0 \%$. El $5,2 \%$ presentó insuficiencia cardíaca Killip $>2$.

El análisis de los eventos intrahospitalarios, así como el de los alejados, se realizó comparando los 3 grupos de riesgo estratificados según el puntaje TIMI. Los resultados se muestran en la Tabla 5, expresados en OR con su intervalo de confianza de 95\% para el grupo de mayor riesgo versus el de riesgo menor. Se puede observar que la mortalidad intrahospitalaria fue más elevada en la medida que
Tabla 4. Porcentaje de procedimientos angiográficos y de revascularización miocárdica efectuados

\begin{tabular}{|lr|}
\hline & $(\%)$ \\
\hline Coronariografía & 52,0 \\
Angioplastia & 24,8 \\
Angioplastia de urgencia & 2,0 \\
Cirugía coronaria & 13,0 \\
Cirugía coronaria de urgencia & 1,7 \\
\hline
\end{tabular}

aumentaba el riesgo, al igual que la incidencia de eventos adversos combinados (muerte, infarto, isquemia recurrente). El análisis de los eventos alejados mostró también que éstos aumentaron en la medida que el puntaje de riesgo inicial era más alto.

El diagnóstico de egreso fue de angina inestable en $59 \%$ de los casos y de infarto del miocardio sin SDST en $37,4 \%$.

\section{DisCUSIÓN}

La información obtenida de registros multicéntricos de pacientes con síndromes coronarios agudos ha sido de gran utilidad para evaluar las características clínicas, los métodos de diagnóstico y los tratamientos utilizados en distintos países 0 regiones del mundo. Hasta la fecha, existe abundante información sobre los síndromes coronarios

Tabla 5. Eventos intrahospitalarios y a los 6 meses para los 3 grupos de riesgo

\begin{tabular}{|ccccc|}
\hline & $\begin{array}{c}\text { Bajo } \\
(\mathrm{n}=179) \\
\%\end{array}$ & $\begin{array}{c}\text { Riesgo } \\
\text { Intermedio } \\
(\mathrm{n}=285) \\
\%\end{array}$ & $\begin{array}{c}\text { Alto } \\
(\mathrm{n}=135)\end{array}$ & OR $(95 \% \mathrm{CI})$ \\
& & & & \\
& & & & \\
\hline Eventos intrahospitalarios & 1,68 & 2,81 & 3,7 & $2,26(0,53-9,61)$ \\
Muerte & 9,5 & 15,1 & 20,0 & $2,38(1,24-4,58)$ \\
Combinado & 0,56 & 8,7 & 25,9 & $62,3(8,4-461,6)$ \\
Insuficiencia cardíaca & 6,2 & 12,3 & 13,3 & $2,35(1,07-5,15)$ \\
Isquemia recurrente & 2,2 & 1,4 & 6,7 & $0,75(0,46-1,21)$ \\
Infarto del miocardio & & & & \\
Eventos a los 6 meses & 0 & 4,5 & 10,9 & $\mathrm{p}=0,003$ \\
Muerte & 15,5 & 18,9 & 31,3 & $2,47(1,08-5,69)$ \\
Combinado & & &
\end{tabular}


agudos con elevación del segmento ST, pero las publicaciones de registros de angina inestable e infarto del miocardio sin elevación del segmento ST son más escasas ${ }^{6-11}$. Algunos de estos registros reportan una realidad nacional, como es el caso de los estudios PRAIS-UK, PEPA y TIMI 37,11,12, y otros reportan resultados de registros internacionales como OASIS, Euro Heart Survey y GRA$\mathrm{CE}^{6,8,9}$. La mayoría de estos registros reflejan una gran heterogeneidad en cuanto a utilización de métodos de diagnóstico y a tratamientos.

En nuestro registro fijamos criterios de inclusión estrictos, de manera que pudiéramos analizar una muestra representativa de angina inestable e infarto SDST. Así, 94\% de nuestros pacientes se presentó con dolor anginoso característico, 72\% presentaba alteraciones de tipo isquémico en el ECG de ingreso y 30\% tuvo elevación de marcadores de daño miocárdico a las $6 \mathrm{~h}$ de ingreso. Las características demográficas, prevalencia de factores de riesgo y antecedentes cardiovasculares previos de nuestra población fueron similares a las de otros registros como ECLA 3, OASIS y GRACE $^{13,6,9}$. Llama la atención en nuestra serie la alta prevalencia de dislipidemia (52\%), similar a la reportada recientemente en el Euro Heart Survey ${ }^{10}$, lo que podría explicarse porque actualmente existe una mayor conciencia médica sobre la necesidad de efectuar determinaciones de lípidos en pacientes que ingresan con síndromes coronarios agudos.

La utilización de terapia antitrombótica muestra un empleo elevado de aspirina (96\%) y heparinas (74\%), concordante con lo reportado en los registros OASIS y PRAIS-UK ${ }^{6,9}$. Es interesante que en nuestra serie se observa un mayor empleo de heparinas de bajo peso molecular en relación a la heparina no fraccionada, lo que también es concordante con las pautas internacionales europeas y norteamericanas ${ }^{1,2}$ y con los datos recientes de los registros GRACE y Euro Heart Survey ${ }^{9,10}$. En cuanto al tratamiento antiisquémico el empleo de betabloqueadores fue cercano al $70 \%$, lo que se compara favorablemente con otros registros internacionales ${ }^{8-10}$.

En nuestra serie se efectuó coronariografía y angioplastia coronaria a 50 y $25 \%$ de los pacientes, respectivamente, lo que es superior a lo reportado por otros registros nacionales como PRAIS-UK y
$\mathrm{PEPA}^{7,11}$ y similar a las cifras de registros internacionales más recientes (52 y $25 \%$ en el Euro Heart Survey, 45 y $21 \%$ en GRACE). Esta conducta es concordante con las recomendaciones más recientes de manejo de los síndromes coronarios agudos, las que favorecen una estrategia invasiva precoz, en base a los resultados de los ensayos clínicos TACTICS y FRISC $2^{14,15}$. El porcentaje relativamente elevado de procedimientos invasivos en esta serie está influido, probablemente, por el hecho de que la mitad de los centros participantes disponía de facilidades para efectuar coronariografía. La ejecución de procedimientos invasivos no guardó relación con el puntaje de riesgo TIMI, lo que también se ha observado en otros registros ${ }^{16}$. En nuestro registro no validamos el impacto de la conducta invasiva sobre la mortalidad.

La estratificación de riesgo, efectuada de acuerdo al score TIMI $^{5}$, nos permitió comprobar que la mayoría de la población de nuestra serie se agrupa en sectores de riesgo intermedio, lo que es muy similar a los hallazgos del registro TIMI $3^{16}$. La necesidad de llevar a cabo una revisión permanente de los criterios de estratificación de riesgo y de su aplicación precoz ha sido destacado en diferentes publicaciones ${ }^{17-19}$.

La mortalidad global en nuestra serie fue baja, $2,6 \%$, lo que es similar a la de otros estudios ${ }^{8-10}$. Sin embargo, tanto la mortalidad como la combinación de eventos adversos se incrementó progresivamente en la medida que el puntaje de riesgo TIMI era más alto, validando así reportes en que se ha aplicado este método en forma retrospectiva en poblaciones seleccionadas de pacientes incluidos en ensayos clínicos ${ }^{20-22}$.

El diagnóstico de egreso fue de angina inestable en $59 \%$ y de infarto sin SDST en 37\%, quedando $3 \%$ de los pacientes sin un diagnóstico definitivo. Estas cifras son también concordantes con otros estudios 9,10 y confirman que la utilización adecuada de los marcadores de daño miocárdico CK o troponinas (basal y a las $6 \mathrm{~h}$ ) permiten identificar una proporción más alta de pacientes con evidencias de necrosis miocárdica, aunque sea mínima.

La limitación más importante de nuestro registro es que no sea un reflejo de la realidad nacional. Es un hecho conocido que en nuestro 
medio existe una baja disponibilidad de camas de unidad coronaria y ello incide en que una proporción alta de pacientes con angina inestable e infarto SDST no tengan diagnóstico o tratamiento oportunos. Nuestra casuística constituye, por lo tanto, una muestra seleccionada de pacientes, que fueron admitidos en centros en los cuales se pudo efectuar diagnóstico y tratamiento adecuados. En $50 \%$ de los centros participantes se contaba con disponibilidad de laboratorio de hemodinamia, lo que se refleja en el alto porcentaje de pacientes estudiados con coronariografía. En todo caso, nuestros resultados demuestran que cuando esto es posible de efectuar la morbimortalidad de los síndromes coronarios agudos puede llegar a ser baja y que la utilización de recursos de diagnóstico y tratamiento se compara favorablemente con la de otros registros internacionales. Por otra parte, la estratificación de riesgo precoz a través de un método sencillo como el puntaje TIMI permite predecir las probabilidades de eventos cardiovasculares adversos, lo cual puede ser de gran utilidad para la toma de decisiones terapéuticas.
LISTADO DE INVESTIGADORES POR CENTRO:

- Hospital Regional de Arica: Dr. Iván Criollo.

- Hospital Regional de La Serena: Dr. Claudio. Bugueño, EUs Patricia Cortés, Patricia Mondaca, Ana María González.

- Hospital Gustavo Fricke Viña del Mar: Dr. Carlos Raffo.

- Hospital Naval de Talcahuano: Dr. Alejandro Dapelo, EU Beatriz Gutiérrez.

- Hospital Regional de Concepción: Dr. Erwin Schumacher.

- Hospital Regional de Temuco: Dr. Benjamín Stockins.

- Hospital Clínico de Valdivia: Dr. Germán Eggers.

- Hospital Regional de Osorno: Dr. Sergio Potthoff.

- Hospital Base de Puerto Montt: Dr. Jaime Venegas, EU María Soledad Hidalgo.

- Hospital de Victoria: Dra. Margarita Figueroa

- Hospital de Quillota: Dra. Minerva Jara.

- Hospital de la Asistencia Pública: Dr. Eduardo Chávez, Dra. Pabla Campos.

- Hospital Clínico de Universidad Católica: Dr. Ramón Corbalán, EU Juanita Rosales.

- Hospital Clínico de Universidad de Chile: Dr. Juan Carlos Prieto, Alum. Daniela Cortez.

- Hospital Dipreca: Dr. Milton Alcaíno, EU María Luisa Zenteno.

- Coordinadora de Centros: Carolina Nazzal.

- Análisis estadístico: Rosa Montaño, Francisco Cumsille.

\section{REFERENCIAS}

1. Bertrand ME, Simoons ML, Fox KAA, Walientin LC, HAMM CW, FADDEN E ET AL. Management of acute coronary syndromes in patients presenting without persistent ST-segment elevation. The Task Force on the Management of Acute Coronary Syndromes of the European Society of Cardiology. Eur Heart J 2002; 23: 1809-40.

2. Braunwald E, Antman EM, Beasley JW, Califf RM, Cheituin MD, Hochman JS et al. ACC/AHA 2002 guideline update for the management of patients with unstable angina and non-ST-segment elevation myocardial infarction: summary

article: a report of the American College of Cardiology/American Heart Association task force on practice guidelines. J Am Coll Cardiol 2002; 40: 1366-74.

3. Corbalán R, Martínez A, Uriarte P, Yovanovich J. Recomendaciones para el diagnóstico y tratamiento de la angina inestable. Rev Chil Cardiol 1999; 18: 31-8.

4. Corbalán R, Martínez A, Uriarte P, Yovanovich J. Recomendaciones para el diagnóstico y tratamiento de la angina inestable. Rev Chil Cardiol 2002; 21: 127-36.

5. Antman EM, Cohen M, Bernink PJ et al. The TIMI risk score for unstable angina/non-ST 
elevation MI: a method for prognostication and therapeutic decision making. JAMA 2000; 284: 835-42.

6. Yusuf S, Flather M, Pogue J, Hunt D, Varigos J, PIEGAS L ET AL. Variations between countries in invasive cardiac procedures and outcomes in patient with suspected unstable angina or myocardial infarction without initial ST elevation. OASIS (Organization to Assess Strategies for Ischaemic Syndromes) Registry Investigators. Lancet 1998; 352: 507-14.

7. CoLinson J, Flather MD, Fox KAA, Findlay I, Rodriguez E, Dooley P et al. Clinical outcomes, risk stratification and practice patterns of unstable angina and myocardial infarction without ST elevation: prospective registry of acute ischaemic syndromes in the UK (PRAIS-UK). Eur Heart J 2000; 21: 1450-7.

8. Fox KAA, Cokkinos DV, Deckers J, Keil U, Magioni A, Steg G et al. The ENACT study: a panEuropean survey of acute coronary syndromes. Eur Heart J 2000; 21: 1440-9.

9. Fox KAA, Goodman SG, Kifin W, Brieger D, Steg PG, DabBous O ET AL. Management of acute coronary syndromes. Variations in practice and outcome. Findings from the Global Registry of Acute Coronary Events (GRACE). Eur Heart J 2002; 23: 1177-89.

10. Hasdai D, Behar S, Walientin L, Danchin N, Gitt AK, Boersma E et aL. A prospective survey of the characteristics, treatments and outcomes of patients with acute coronary syndromes in Europe and the Mediterranean basin. The Euro Heart Survey of Acute Coronary Syndromes (Euro Heart Survey ACS). Eur Heart J 2002; 23: 1190-201.

11. López de Sá E, López-Sendón J, Anguera I, Bethencourt A, Bosch X. Prognostic value of clinical variables at presentation in patients with non-STsegment elevation acute coronary syndromes. Results of the Proyecto de Estudio del Pronóstico de la Angina (PEPA). Medicine (Baltimore) 2002; 81: 434-42.

12. Stone PH, Thompson B, Anderson HV, KronenBerg MW, Gibson RS, Rogers WJ et aL, For the TIMI III Registry Study Group. Influence of race, sex, and age on management of unstable angina and non-Q-wave myocardial infarction: the TIMI III Registry. JAMA 1996; 275: 1104-12.
13. Bazzino O, Díaz R, Tajer C, Paviotti C, Meie E, Trivi M et al, For the ECLA CoLaborative Group Buenos Aires, ARgentina. Clinical predictors of in hospital prognosis in unstable angina (ECLA 3). Am Heart J 1999; 137: 322-31.

14. Cannon CP, Weintraub WS, Demopoulos LA, Vicari R, FREY MJ, LaKKIS N ET AL. Comparison of early invasive and conservative strategies in patients with unstable coronary syndromes treated with the glycoprotein IIb/IIIa inhibitor tirofiban. N Engl J Med 2001; 344: 1879-87.

15. FrisC II INVESTIGATORS: Invasive compared with non invasive treatment in unstable coronary disease: Frisc II prospective randomized multicentre study. Fragmin and Fast Revascularization during instability in coronary artery disease Investigators. Lancet 1999; 354: 708-15.

16. Scirica BM, Cannon CP, Antman EM, Murphy SA, Morrow DA, Sabatine MS et al. Validation of the thombolysis in myocardial infarction (TIMI) risk score for unstable angina pectoris and non-STelevation myocardial infarction in the TIMI III registry. Am J Cardiol 2002; 90: 303-5.

17. Antman EM, Corbalán R, Huber K, JafFe AS. Issues in early risk stratification for UA/NSTEMI. Eur Heart J 2001; 3 (Suppl J): J6-J14.

18. Antman EM, Tanasijevic MJ, Thompson B. Cardiacspecific troponin I levels to predict the risk of mortality in patients with acute coronary syndromes. N Engl J Med 1996; 335: 1342-9.

19. Myocardial infarction ReDEFInED. A consensus document of the Joint European Society of Cardiology/American College of Cardiology Committee for the redefinition of myocardial infarction. J Am Coll Cardiol 200??; 36: 959-69.

20. Morrow DA, ANtman EM, Snapinn S. An Integrated Clinical Approach to Predicting the benefit of Tirofiban in Non-ST Elevation Acute Coronary Syndromes: Application of the TIMI Risk Score for UA/ NSTEMI in PRISM-PLUS. Eur HeartJ 2002; 23: 223-9.

21. Yusuf S, Zhao F, Mehta SR, Chrolavicius S, Tognoni G, Fox KK. Effects of clopidogrel in addition to aspirin in patients with acute coronary syndromes without ST-segment elevation. N Engl J Med 2001; 345: 494-502.

22. Cannon CP, Weintraub WS, Demopoulos LA. Comparison of early invasive and conservative strategies in patients with unstable coronary syndromes treated with the glycoprotein Iib/Lل inhibitor tirofiban. N Engl J Med 2001; 344: 1879-87. 